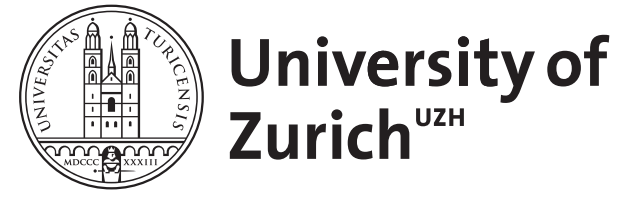

\title{
Diagnostik von Pilzinfektionen
}

Bosshard, Philipp P

DOI: https://doi.org/10.1024/0040-5930/a000817

Posted at the Zurich Open Repository and Archive, University of Zurich ZORA URL: https://doi.org/10.5167/uzh-130098

Journal Article

Accepted Version

Originally published at:

Bosshard, Philipp P (2016). Diagnostik von Pilzinfektionen. Therapeutische Umschau. Revue thérapeutique, 73(8):449-455.

DOI: https://doi.org/10.1024/0040-5930/a000817 


\section{Dermatologische Klinik, Universitätsspital Zürich \\ Diagnostik von Pilzinfektionen \\ Philipp P. Bosshard}

\section{Zusammenfassung}

Die klinischen Zeichen einer Pilzinfektion sind häufig unspezifisch und es gibt meist viele mögliche Differentialdiagnosen. Laboruntersuchungen sind für die Diagnose deshalb essentiell. Für eine optimale Untersuchung ist die Präanalytik wichtig, es braucht eine korrekte Probenentnahme und genügend Informationen auf dem Laborauftrag. Die Methoden umfassen Mikroskopie, Kultur, Serologie (Biomarker) und Molekularbiologie. Je nach Mykose und je nach Infektionsort werden andere Methoden gewählt. In den letzten Jahren haben neue Entwicklungen bei serologischen und molekularbiologischen Methoden die Diagnostik erheblich verbessert und erlauben z. T. die Diagnosestellung, wo dies früher nicht möglich gewesen wäre.

\section{Einleitung}

Wie bei anderen mikrobiellen Infekten beruht die Diagnose eines Pilzinfekts auf einer Kombination von klinischer Untersuchung und Laboruntersuchung. Einige der oberflächlichen Mykosen präsentieren sich i.d.R. mit typischen Läsionen (Bsp. Pityriasis versicolor), andere haben ein breites Spektrum klinischer Präsentationen (Bsp. Tinea corporis). Erschwerend kommt hinzu, dass es gerade bei vielen oberflächlichen Mykosen eine mehr oder weniger lange Liste an dermatologischen Differentialdiagnosen gibt. So muss man bei Pityriasis versicolor auch an Vitiligo, Pityriasis alba, Pityriasis rosea, seborrhoische Dermatitis oder auch mal an eine sekundäre Syphilis denken. Und bei einer Tinea corporis kommen differentialdiagnostisch auch ein nummuläres Ekzem, atopische Dermatitis, Parapsoriasis, Psoriasis, subakuter Lupus erythematodes oder Impetigo in Frage. Bei tiefen oder invasiven Pilzinfektionen sind die klinischen Symptome meist unspezifisch und können auch von anderen Infektionen, von der Grunderkrankung oder von anderen Komplikationen herrühren. Laboruntersuchungen sind deshalb für die Diagnose eines Pilzinfekts essentiell.

Zwei wichtige Säulen der Pilzdiagnostik sind Direktmikroskopie und Kultur (Abb. 1). Bei invasiven Mykosen kommen aber serologische (Biomarker) und molekularbiologische Methoden vermehrt zum Einsatz und sind z.T. akkurater als die klassischen Methoden. Die Diagnostik ist entscheidend von der Qualität des eingesandten Materials 
(Entnahmeort und -technik, Lagerung und Versand) und von den klinischen Angaben abhängig. Dies ist sozusagen das Fundament auf dem die Diagnostik aufbaut. Im folgenden Kapitel wird deshalb zuerst auf Besonderheiten der Präanalytik eingegangen. Anschliessend werden Aspekte der verschiedenen Labormethoden erläutert.

\section{Präanalytik}

In Tabelle 1 sind die wichtigsten Punkte zusammengefasst.

Probenentnahme und-transport nicht-dermatologischer Proben:

Mit Ausnahme bei den dermatologischen Proben gelten im Allgemeinen dieselben Richtlinien wie für bakteriologische Untersuchungen. Grundsätzlich so viel natives Material wie möglich vom Infektionsort in einem sterilen Gefäss geschickt werden. Bei Liquor sollten mindestens $2 \mathrm{ml}$ für eine bakteriologische und mykologische Untersuchung abgenommen werden. Für Blutkulturen gelten grundsätzlich dieselben Regeln wie für den Nachweis von Bakterien [1-3]. Es gibt allerdings Hinweise, dass die Verwendung von speziellen Pilzkulturflaschen die Sensitivität erhöhen kann [1, 2].

\section{Probenentnahme und-transport dermatologischer Proben:}

Hier gilt, i) Läsion/Entnahmestelle wenn möglich vorher mit $70 \%$ Alkohol reinigen, ii) bei einer Nagelmykose Nagelspäne und mürbes, subunguales Nagelmaterial so weit wie möglich proximal entnehmen, iii) bei Tinea corporis Schuppen aus dem Randbereich der kutanen Läsion abkratzen, iv) bei Tinea capitis Schuppen und epilierte Haar mit Haarwurzel entnehmen und v) Proben trocken lagern und mit den dafür vorgesehenen Versandpackungen (z.B. Dermapak) versenden.

\section{Laborauftrag und zusätzliche Angaben:}

Essentiell ist, dass der Pilznachweis explizit verlangt wird und dass zusätzlich Angaben gemacht werden. Die einsenden Ärzte sind gebeten, die Verdachtsdiagnose und Informationen wie Reiseanamnese, Grundkrankheiten, Tierkontakte etc. mitzuteilen. Diese Informationen sind wichtig für die korrekte Verarbeitung der Proben und für die Interpretation der Resultate. Ganz wichtig sind z.B. den Verdacht auf eine primäre Systemmykose (dimorphe Pilze) zu äussern oder anzugeben, dass der Patient mit Tinea ein Landwirt ist, weil dies jeweils andere Kulturmedien und eine längere Bebrütung zur Folge hat [4]. Auch Hinweise auf Tropenaufenthalte sind wesentlich, um das dann wesentlich erweiterte Erregerspektrum zu berücksichtigen und allenfalls entsprechende Zusatzuntersuchungen in die Wege zu leiten. 


\section{Mikroskopie}

Direktmikroskopie:

Die Direktmikroskopie ist bei jeder mykologischen Untersuchung durchzuführen. Es ist ein günstiges und schnelles Verfahren von eminenter Bedeutung. Es stehen verschiedene Methoden zur Verfügung. Bei den Nativpräparaten wird das Material flüssig (meist unter Zugabe eines Aufhellers oder einfachen Farbstoffs) untersucht. Bei gefärbten Präparaten wird das Material vorher auf einem Objektträger fixiert und anschliessend mehr oder weniger aufwändig gefärbt. Tabelle 2 gibt einen Überblick über die häufig verwendeten Methoden in der Mikroskopie. In Abbildung 2 sind verschiedene Beispiele positiver Präparate dargestellt.

Ein grosser Vorteil der Mikroskopie ist, dass ein erstes Resultat innerhalb weniger Minuten vorliegen kann (s. Tabelle 2). Generell kann gesagt werden, dass der Nachweis von Schimmelpilzhyphen immer relevant ist, wohingegen die Interpretation beim Nachweis von Hefezellen Material- und Mengen-abhängig ist. In manchen Fällen ist das Resultat der Direktmikroskopie diagnosestellend. Beispiele hierfür sind der Nachweis von intrazellulären Histoplasma capsulatum Hefezellen im Knochenmark, Coccidioides sp. Sphaerulen im Gewebe, Pneumocystis jirovecii Zysten im respiratorischen Material oder typischen Malassezia Pilzelementen bei Pityriasis versicolor. In anderen Fällen kann die Direktmikroskopie wegweisend für die Therapie sein. Beispielsweise hat der Nachweis von Schimmelpilzhyphen in einer bronchoalveolären Lavage eines Neutropenikers umgehend therapeutische Konsequenzen. Ferner hilft die mikroskopische Untersuchung bei der Beurteilung eines später in der Kultur gewachsenen Pilzes.

Einschränkend muss erwähnt werden, dass die Sensitivität der Direktmikroskopie etwas geringer ist als die der Kultur [5]. Ein negatives Resultat schliesst eine Pilzinfektion nie aus. Umgekehrt wird auch von falsch-positiven Resultaten berichtet. Hierfür kann insbesondere die mangelnde Erfahrung des Untersuchers verantwortlich sein.

\section{Histopathologie:}

Die histopathologische Untersuchung am Gewebeschnitt ist eine geeignete Methode für die Diagnose einer subkutanen oder tiefen Mykose. In der Hämatoxylin-Eosin-Färbung lassen sich Pilzstrukturen schlecht darstellen, deshalb wird empfohlen bei Verdacht zusätzliche Färbungen zu machen. Meistens werden die PAS (Periodic Acid Schiff) und die Silberfärbung (Grocott) verwendet. 


\section{Kultur und Identifikation}

Kultur:

Die Kultur nimmt nach wie vor einen wichtigen Stellenwert ein, da sie es erlaubt, den Erreger auf Speziesebene zu identifizieren und allenfalls eine Resistenzprüfung durchzuführen. Die Nährmedien werden entsprechend dem klinischen Material gewählt. Häufig kommen mehrere Medien zum Einsatz; allgemeine Pilzmedien (mit oder ohne Antibiotikazusatz) und selektive Pilzmedien (z.B. mit Cycloheximid). Die Inkubation (Zeitdauer und Temperatur) ist abhängig davon, welche Pilze erwartet werden (s. Abb. 3). Allgemein kann gesagt werden, dass für den Nachweis von Hefen (Blutkulturen, Schleimhaut, Urin) 5-7 Tage inkubiert werden soll, für den Nachweis von Hefen oder Schimmelpilzen (respiratorische Proben, Augenproben, Wunden, Abszesse) 14 Tage, für den Nachweis von Dermatophyten 4 Wochen und für den Nachweis von dimorphen Pilzen 6 Wochen [4].

Hefen wachsen meist relativ gut auf den Kulturmedien. Die Sensitivität von Blutkulturen bei Candidämie liegt im Bereich von 50-75 \%[3]. Im Fall invasiver Aspergillosen ist bekannt, dass Blutkulturen meist negativ bleiben und Kulturen von bronchoalveolären Lavagen (BAL) eine Sensitivität von bis zu 60 \% [6] haben. Es muss auch bedacht werden, dass es bei einer positiven Kultur nicht immer möglich ist, zwischen Kontamination, Besiedlung und Infektionserreger zu unterscheiden. Dies gilt insbesondere dann, wenn ein opportunistischer Pilz wie Candida albicans oder Aspergillus fumigatus aus einem normalerweise nicht-sterilem Material isoliert wird. In solchen Situationen muss die Menge an gewachsenen Kolonien und das Resultat der Direktmikroskopie und/oder anderer Methoden (s. serologische und molekularbiologische Methoden) berücksichtigt werden.

Noch einmal soll betont werden, wie wichtig zusätzlich Angaben wie Verdachtsdiagnose und Informationen wie Reiseanamnese, Grundkrankheiten, Tierkontakte etc. bei der Auftragserteilung sind. Der Verdacht auf eine primäre Systemmykose (dimorphe Pilze) muss beispielsweise unbedingt mitgeteilt werden; dies beeinflusst die Inkubationsdauer und auch die Sicherheitsvorkehrungen im Labor (Pilze der Risikogruppe 3 müssen unter speziellen Sicherheitsvorkehrungen bearbeitet werden). Auch die Information „Landwirt“ bei einem Verdacht auf Tinea ist wichtig, weil hier zusätzliche Medien und eine längere Bebrütung gewählt werden [4].

Identifikation: 
Pilze wie Candida albicans und Aspergillus fumigatus wachsen relativ schnell und gut und die Kolonien können meist innerhalb von zwei Tagen identifiziert werden. Die Identifikation kultureller Isolate, insbesondere von Hefen, hat in den letzten Jahren grosse Fortschritte gemacht. Mit MALDI-TOF werden Kulturisolate innerhalb von wenigen Minuten identifiziert. Bei Hefen liegt die Identifizierungsrate in den meisten grösseren Studien >95 \% [7]. Die Identifikation von Schimmelpilzen und Dermatophyten ist nach wie vor anspruchsvoll; die klassische Identifikation mittels Makro- und Mikromorphologie ist zeitaufwändig und braucht erhebliche Erfahrung. Abbildung 4 zeigt illustrativ die Kultur und ein mikroskopische Präparat eines Schimmelpilzes und eines Dermatophyten. Auch die Untersuchung mit MALDI-TOF ist aufwändiger als bei Hefen und Bakterien (die Proteine müssen zuerst extrahiert werden) und komplexer, da Schimmelpilze innerhalb einer Kolonie ganz unterschiedliche Morphologien aufweisen, was sich direkt im Proteinspektrum niederschlägt. Zudem sind die zurzeit erhältlichen kommerziellen Datenbanken noch sehr limitiert was Schimmelpilze angeht. Entsprechend ist die Identifizierungsrate in verschiedenen Studien ziemlich unterschiedlich und liegt zwischen 72 und 99 \% [7]. Ähnlich ist es bei Dermatophyten; hier ist die Identifizierungsrate meist $>85 \%$ [7, 8]. Führen MALDI-TOF und/oder konventionelle Identifikation nicht zum Ziel, kann im Bedarfsfall in einem Labor mit der entsprechenden Expertise eine DNASequenzierung durchgeführt werden $[9,10]$.

\section{Molekularbiologischer Direktnachweis}

Es gibt eine schier unüberblickbare Anzahl von auf der Polymerasekettenreaktion (PCR) beruhenden Systemen zum Nachweis von Pilz-DNA bei invasiven und auch nichtinvasiven Mykosen. Manche Laboratorien haben eigene Methoden entwickelt. Unterdessen gibt es aber eine grosse Palette von kommerziellen Produkten. Für die Laboratorien werden auch erste Ringversuche angeboten. Ein Vorteil des molekularen Direktnachweises ist, dass das Resultat i.d.R. innerhalb von 24 Std. vorliegt. Ein wichtiger Nachteil ist die hohen Kosten. Tabelle 3 gibt einen Überblick über die gängigen Verfahren.

In einer Cochrane Analyse [11] wurde kürzlich die Wertigkeit der PCR aus Blut zum Nachweis einer invasiven Aspergillose bei immunsupprimierten Patienten untersucht. 18 Studien mit total 1765 Patienten mit hohem Risiko für eine invasive Aspergillose wurden eingeschlossen. Die Prävalenz war durchschnittlich 12 \%. Der Grossteil der Patienten erhielt eine Chemotherapie wegen maligner hämatologischer Erkrankung oder war Knochenmark-transplantiert. Wurde ein einmalig positives Ergebnis als Kriterium festgelegt, waren die durchschnittliche Sensitivität und Spezifität der PCR $80.5 \%$ und 
$78.5 \%$. Wurden zwei aufeinanderfolgende positive Resultate gefordert, sank die Sensitivität auf $58 \%$, die Spezifität stieg dafür auf $96.2 \%$. Die Konklusion aus dieser Analyse ist, dass zwei aufeinanderfolgende positive Resultate gefordert werden müssen, da sonst bei der tiefen Prävalenz der Erkrankung zu viele falsch-positive Befunde entstehen. Mit diesem Schema hat der Test einen hohen negativen prädiktiven Wert, d.h. bei negativem Resultat kann eine invasive Aspergillose ausgeschlossen werden. Es wurde auch wiederholt gezeigt, dass die PCR aus BAL auch sinnvoll sein kann (Sensitivität 79 \%, Spezifität 94 \%) [12].

Bei Patienten mit wahrscheinlicher oder erwiesener invasiver Candidiasis hat eine Metaanalyse mit 17 Studien eine Sensitivität und Spezifität der PCR aus Blut von 93 \% und $95 \%$ gezeigt [13]. Der kommerzielle SeptiFast Testkit hatte in einer anderen Metaanalyse eine Sensitivität und Spezifität von 61 \% und 99 \% für Fungämien [14]. Kürzlich wurde ein neuer Test, der T2Candida Panel, mit vielversprechenden Resultaten lanciert [15]. Auch für den Nachweis von Dermatophyten sind in-house und kommerzielle molekulare Methoden vorhanden. Die Sensitivität verschiedener Studien liegt zwischen 80 $\%$ und $100 \%[16]$.

\section{Serologische Methoden (Biomarker)}

In der medizinischen Mykologie sind verschiedene serologische Tests etabliert. Tabelle 3 gibt einen Überblick über die gängigen Verfahren. Bei vielen davon werden Antigene (Biomarker) nachgewiesen, bei anderen werden Antikörper gesucht.

\section{Galaktomannan:}

Dieser Test wird bei Risikopatienten zur Diagnose einer invasiven Aspergillose verwendet. Galaktomannan ist ein Zellwandbestandteil von Aspergillus sp., der während des invasiven Wachstums im Serum und anderen Flüssigkeiten wie Urin und BAL (bronchoalveoläre Lavage) mittels eines kommerziell erhältlichen ELISAs nachgewiesen werden kann. In einer Cochrane Analyse [17] wurde die diagnostische Wertigkeit aus Serum bei neutropenischen Patienten ermittelt (s. Tabelle 2); die Sensitivität und Spezifität sind abhängig vom gewählten Grenzwert (cut-off). Das Labor und die Kliniker müssen sich im Klaren sein, dass wenn die Sensitivität gesteigert wird (cut-off 0.5) die Spezifität tiefer wird. Das hat zur Folge, dass mehr falsch-positive als echt-positive Resultate entstehen; in der erwähnten Analyse war die Prävalenz invasiver Aspergillose bei den neutropenischen Risikopatienten $11 \%$. Mit einer Sensitivität von 78 \% (Grenzwert 0.5) werden bei 100 untersuchten Patienten zwei der elf Patienten mit invasiver Aspergillose verpasst. Wegen der tiefen Spezifität von $85 \%$ werden aber 13 Patienten ein 
falsch-positives Ergebnis haben und entweder unnötig therapiert werden oder weitere Untersuchungen (z.B. CT) erhalten. Mit einem Grenzwert von 1.5 würden vier von elf Patienten mit invasiver Aspergillose verpasst und es gäbe nur sechs falsch-positive Ergebnisse.

Von grosser Bedeutung und in mehreren Studien gezeigt ist, dass der GalaktomannanTest bei 40-70 \% der Patienten durchschnittlich circa eine Woche (0-24 Tage) früher positiv anzeigt als der klinische Verdacht auf eine invasive Aspergillose.

Dementsprechend wird empfohlen, den Test 2-3x pro Woche als Screening einzusetzen. Des Weiteren konnte gezeigt werden, dass die Sensitivität und Spezifität aus BAL höher sind als aus Serum zum Nachweis einer pulmonalen Aspergillose [18].

\section{Beta Glukan:}

1,3-beta-D-Glukan ist ein Zellwandbestandteil von Candida sp., Aspergillus sp. und anderen Pilzen wie Fusarium sp. oder Pneumocystis jirovecii, nicht aber von Zygomyceten und Kryptokokken. Dementsprechend braucht es bei einem positiven Resultat weitere Untersuchungen, um das infektiöse Agens zu identifizieren. Sensitivität und Spezifität sind in etwa vergleichbar mit den anderen Biomarkern. Es wird empfohlen, bei Risikopatienten den Test 2x pro Woche anzuordnen. Bei diesem Schema eignet sich der Test insbesondere, um eine invasive Pilzinfektion auszuschliessen. Falsch-positive Resultate können u.a. auftreten bei Hämodialyse, Gram-negativer Sepsis oder Antibiotikatherapie (Amoxicillin-Clavulansäure, Piperacillin-Tazobactam).

\section{Candida Mannan und Antimannan:}

Mannan ist ein Zellwandbestandteil von Candida sp. Der kombinierte Nachweis von Mannan (Antigen) und Anti-Mannan (Antikörper) gilt als sensitiver und spezifischer Nachweis für eine invasive Candidiasis. Es ist wichtig, nicht einen Test alleine durchzuführen. Die Sensitivität ist Spezies-abhängig und höher für $C$. albicans, $C$. glabrata und C. tropicalis (80-100 \%) als für C. krusei und C. parapsilosis (40-50\%). Auch für diesen kombinierten Test wird empfohlen, inn bei Risikopatienten wiederholt durchzuführen. Besonders wichtig zu erwähnen ist, dass der Test in Studien bei rund 70 $\%$ der Patienten mit Candidämie im Durchschnitt eine Woche früher positiv war als die Blutkulturen und bei rund $85 \%$ der Patienten mit invasiver Candidiasis mit Befall von Leber und Milz sogar zwei Wochen früher positiv als die Bildgebung und so eine frühe Therapie erlaubte [19].

Weitere serologische Methoden: 
Der Vollständigkeit halber wird hier erwähnt, dass es für die Diagnostik einer

Kryptokokkose sensitive Antigen-Tests zum Nachweis aus Serum, Liquor und Urin gibt und zur Diagnose einer primären Systemmykose (Histoplasmose, Kokzidioidomykose, Blastomykose, Parakokzidioidomykose, Penicillose) stehen sowohl Antigen- als auch Antikörper-Nachweisverfahren zur Verfügung, auf die hier nicht weiter eingegangen wird. Zum serologischen Nachweis der im Zunehmen begriffenen und mit einer hohen Mortalität einhergehenden Mucormykose gibt es leider noch keine Produkte.

\section{Laboratory Diagnosis of fungal infection}

Clinical presentations of fungal infections are often non-specific and can be caused by a wide range of diseases. Therefore, laboratory tests are needed for diagnosis. For optimal investigation clinicians should provide the laboratory with adequate specimens and sufficient background information. The methods in the laboratory encompass microscopy, culture, serology (biomarkers) and molecular diagnosis. Appropriate methods differ from mycosis to mycosis as well as the site of infection. The direct microscopic examination is a fast, simple and useful method and should be done on every specimen where possible. Isolation in culture will permit identification of most pathogenic and resistance testing if necessary. In recent years, new developments in serological and molecular methods have improved the diagnostics substantially and allow diagnosis in cases where this previously was not possible. For example for invasive candidiasis and invasive aspergillosis both biomarkers and molecular tests on serum or other sample material are available which outweigh traditional diagnostics in terms of sensitivity and specificity.

\section{Referenzen}

1. Arendrup MC, Boekhout T, Akova M, et al. ESCMID and ECMM joint clinical guidelines for the diagnosis and management of rare invasive yeast infections. Clin Microbiol Infec 2014;20:76-98.

2. Baron EJ, Miller JM, Weinstein MP, et al. A Guide to Utilization of the Microbiology Laboratory for Diagnosis of Infectious Diseases: 2013 Recommendations by the Infectious Diseases Society of America (IDSA) and the American Society for Microbiology (ASM). Clinical Infectious Diseases 2013;57(4):E22-E121.

3. Cuenca-Estrella M, Verweij PE, Arendrup MC, et al. ESCMID* guideline for the diagnosis and management of Candida diseases 2012: diagnostic procedures. Clin Microbiol Infec 2012;18:9-18.

4. Bosshard PP. Incubation of fungal cultures: how long is long enough? Mycoses 2011;54(5):E539-E45.

5. Merz WG, Roberts GD. Algorithms for detection and identification of fungi. In: Murray PR, Baron EJ, Jorgensen ML, Pfaller MA, Yolken RH, eds. Manual of clinical microbiology. 2. 8th ed. Washington, D.C.: American Society for Microbiology; 2003:166885. 
6. Richardson MD, Warnock DW. Fungal infection: diagnosis and management. 3rd ed. Malden, MA: Blackwell Publishing; 2003.

7. Cassagne C, Normand AC, L'Ollivier C, Ranque S, Piarroux R. Performance of MALDI-TOF MS platforms for fungal identification. Mycoses 2016.

8. de Respinis S, Tonolla M, Pranghofer S, Petrini L, Petrini O, Bosshard PP. Identification of dermatophytes by matrix-assisted laser desorption/ionization time-of-flight mass spectrometry. Med Mycol 2013;51(5):514-21.

9. Ciardo DE, Schar G, Altwegg M, Bottger EC, Bosshard PP. Identification of moulds in the diagnostic laboratory--an algorithm implementing molecular and phenotypic methods. Diagn Microbiol Infect Dis 2007;59(1):49-60.

10. Ciardo DE, Schar G, Bottger EC, Altwegg M, Bosshard PP. Internal transcribed spacer sequencing versus biochemical profiling for identification of medically important yeasts. J Clin Microbiol 2006;44(1):77-84.

11. Cruciani M, Mengoli C, Loeffler J, et al. Polymerase chain reaction blood tests for the diagnosis of invasive aspergillosis in immunocompromised people. Cochrane Database Syst Rev 2015;10:CD009551.

12. Tuon FF. A systematic literature review on the diagnosis of invasive aspergillosis using polymerase chain reaction (PCR) from bronchoalveolar lavage clinical samples. Rev Iberoam Micol 2007;24(2):89-94.

13. Avni T, Leibovici L, Paul M. PCR diagnosis of invasive candidiasis: systematic review and meta-analysis. J Clin Microbiol 2011;49(2):665-70.

14. Chang SS, Hsieh WH, Liu TS, et al. Multiplex PCR system for rapid detection of pathogens in patients with presumed sepsis - a systemic review and meta-analysis. PLoS One 2013;8(5):e62323.

15. Mylonakis E, Clancy CJ, Ostrosky-Zeichner L, et al. T2 magnetic resonance assay for the rapid diagnosis of candidemia in whole blood: a clinical trial. Clin Infect Dis 2015;60(6):892-9.

16. Jensen $\mathrm{RH}$, Arendrup MC. Molecular diagnosis of dermatophyte infections. Curr Opin Infect Dis 2012;25(2):126-34.

17. Leeflang MM, Debets-Ossenkopp YJ, Wang J, et al. Galactomannan detection for invasive aspergillosis in immunocompromised patients. Cochrane Database Syst Rev 2015;12:CD007394.

18. Guo YL, Chen YQ, Wang K, Qin SM, Wu C, Kong JL. Accuracy of BAL galactomannan in diagnosing invasive aspergillosis: a bivariate metaanalysis and systematic review. Chest 2010;138(4):817-24.

19. Marchetti O, Lamoth F, Mikulska M, et al. ECIL recommendations for the use of biological markers for the diagnosis of invasive fungal diseases in leukemic patients and hematopoietic SCT recipients. Bone Marrow Transplant 2012;47(6):846-54.

20. Karageorgopoulos DE, Vouloumanou EK, Ntziora F, Michalopoulos A, Rafailidis PI, Falagas ME. beta-D-glucan assay for the diagnosis of invasive fungal infections: a meta-analysis. Clin Infect Dis 2011;52(6):750-70.

21. Perfect JR, Bicanic T. Cryptococcosis diagnosis and treatment: What do we know now. Fungal Genet Biol 2015;78:49-54.

22. Huang HR, Fan LC, Rajbanshi B, Xu JF. Evaluation of a new cryptococcal antigen lateral flow immunoassay in serum, cerebrospinal fluid and urine for the diagnosis of cryptococcosis: a meta-analysis and systematic review. PLoS One 2015;10(5):e0127117. 23. Wheat LJ. Antigen detection, serology, and molecular diagnosis of invasive mycoses in the immunocompromised host. Transpl Infect Dis 2006;8(3):128-39.

\section{Korrespondenzadresse}

Dr. Philipp P. Bosshard

Dermatologische Klinik 
Universitätsspital Zürich

Gloriastrasse 31

8091 Zürich

philipp.bosshard@usz.ch

Tel + 41442553972

Fax +41442554418 
Tabelle 1: Präanalytik Merkpunkte

\begin{tabular}{|c|c|}
\hline $\begin{array}{l}\text { Proben (nicht- } \\
\text { dermatologisch) }\end{array}$ & $\begin{array}{l}\text { - Generell gleich wie für bakteriologische Untersuchung } \\
\text { - Blutkultur: Pilzblutkulturflasche in Erwägung ziehen } \\
\text { - Liquor: } \geq 2 \mathrm{ml}\end{array}$ \\
\hline Dermatologische Proben & $\begin{array}{l}\text { - } \text { Entnahmestelle mit } 70 \text { \% Alkohol desinfizieren } \\
\text { - Nagelspäne und subunguales Material möglichst } \\
\text { proximal entnehmen } \\
\text { - Hautschuppen aus dem Randbereich der Läsion } \\
\text { - Haare mit Wurzel } \\
\text { - Proben trocken lagern und verschicken }\end{array}$ \\
\hline Laborauftrag & $\begin{array}{l}\text { - Pilznachweis verlangen } \\
\text { - Verdachtsdiagnose angeben } \\
\text { - Informationen wie Reiseanamnese, Grundkrankheiten, } \\
\text { Tierkontakt angeben }\end{array}$ \\
\hline
\end{tabular}


Tabelle 2: Häufig verwendeten Methoden der Direktmikroskopie

\begin{tabular}{|c|c|c|c|}
\hline Methode & Verwendung & Zeitbedarf & Bemerkung \\
\hline \multicolumn{4}{|l|}{ Nativpräparate: } \\
\hline Kalilauge (KOH), 10-20 \% & $\begin{array}{l}\text { Nachweis von Pilzen aus fast allen } \\
\text { Proben. } \\
\text { Aufhellung der Probe }\end{array}$ & $5 \mathrm{~min}$ & $\begin{array}{l}\text { Sehr schnell. Speziell geeignet im } \\
\text { Phasenkontrast }\end{array}$ \\
\hline $\begin{array}{l}\text { Kongorot in Natriumdodecylsulfat } \\
\text { (SDS) }\end{array}$ & $\begin{array}{l}\text { Bei dermatologischen Proben } \\
\text { bewährt }\end{array}$ & $10-20 \mathrm{~min}$ & Sehr schnell \\
\hline Fluorochrom-Farbstoff & $\begin{array}{l}\text { Nachweis von Pilzen mit } \\
\text { fluoreszierenden Farbstoffen wie } \\
\text { Calcofluor oder Blankophor }\end{array}$ & $2 \min$ & Braucht ein Fluoreszenzmikroskop \\
\hline Tuschepräparat & $\begin{array}{l}\text { Nachweis von Kryptokokken im } \\
\text { Liquor }\end{array}$ & $2 \min$ & $\begin{array}{l}\text { Sensitivität dem Antigenschnelltest } \\
\text { unterlegen }\end{array}$ \\
\hline \multicolumn{4}{|l|}{ Gefärbte Präparate: } \\
\hline Grampräparat & Nachweis von Bakterien & $5 \mathrm{~min}$ & $\begin{array}{l}\text { Hefen (ausser Kryptokokken) } \\
\text { werden gut angefärbt. } \\
\text { Schimmelpilze färben z.T. sehr } \\
\text { schwach an }\end{array}$ \\
\hline PAS (Periodic Acid Schiff) & Nachweis von Pilzen & $60 \mathrm{~min}$ & Alle Pilzelemente anfärbbar \\
\hline Silberfärbung (Grocott) & Nachweis von Pilzen & $60 \mathrm{~min}$ & $\begin{array}{l}\text { Aufwändige aber beste Darstellung } \\
\text { von Pilzelementen. Nur in } \\
\text { Spziallabors verfügbar }\end{array}$ \\
\hline
\end{tabular}


Tabelle 3: Molekularbiologische und serologische Methoden (Biomarker) mit Testeigenschaften

\begin{tabular}{|c|c|c|c|c|c|c|}
\hline Test & Erreger & Material & Sensitivität & Spezifität & Bemerkung & Referenz \\
\hline PCR & Aspergillus & $\begin{array}{l}\text { Serum, } \\
\text { BAL }\end{array}$ & $\begin{array}{l}\text { Serum 1x: } 81 \%{ }^{*} \\
\text { Serum 2x: } 58 \%{ }^{*} \\
\text { BAL: } 79 \%\end{array}$ & $\begin{array}{l}\text { Serum 1x: } 79 \%{ }^{*} \\
\text { Serum 2x: } 96 \%{ }^{*} \\
\text { BAL: } 94 \%\end{array}$ & $\begin{array}{l}\text { Sensitivität und Spezifität davon } \\
\text { abhängig, ob eine oder zwei } \\
\text { aufeinanderfolgende positive } \\
\text { Proben gefordert werden. }\end{array}$ & {$[11,12]$} \\
\hline PCR & Candida & Serum & $\begin{array}{l}\text { In-house: 82-98 \% } \\
\text { Kommerziell: } 61-91 \%\end{array}$ & $\begin{array}{l}\text { In-house: 87-98 \% } \\
\text { Kommerziell: 94-99 \% }\end{array}$ & $\begin{array}{l}\text { Kommerzielle Tests sind der } \\
\text { SeptiFast und der neue } \\
\text { T2Candida Panel. }\end{array}$ & [13-15] \\
\hline PCR & Dermatophyten & $\begin{array}{l}\text { Nagel, } \\
\text { Haut, } \\
\text { Haare }\end{array}$ & $80-100 \%$ & - & $\begin{array}{l}\text { Sensitiver und schneller als die } \\
\text { Kultur. }\end{array}$ & [16] \\
\hline Galaktomannan & Aspergillus & $\begin{array}{l}\text { Serum, } \\
\text { BAL }\end{array}$ & $\begin{array}{l}\text { Serum, cut-off 0.5: } 82 \% \\
\text { Serum, cut-off 1.5: } 61 \% \\
\text { BAL: } 85-92 \%\end{array}$ & $\begin{array}{l}\text { Serum, cut-off 0.5: } 81 \% \\
\text { Serum, cut-off 1.5: } 93 \% \\
\text { BAL: } 94-98 \%\end{array}$ & $\begin{array}{l}\text { Sensitivität und Spezifität sind } \\
\text { vom Grenzwert (cut-off) } \\
\text { abhängig. Optimaler Grenzwert } \\
\text { unklar. }\end{array}$ & {$[17,18]$} \\
\hline Beta Glukan & $\begin{array}{l}\text { Candida, } \\
\text { Aspergillus, } \\
\text { Fusarium, } \\
\text { Pneumocystis } \\
\text { jirovecii }\end{array}$ & Serum & $\begin{array}{l}\text { Candida: }>65 \% \\
\text { Aspergillus: } 76 \%\end{array}$ & $\begin{array}{l}\text { Candida: }>80 \% \\
\text { Aspergillus: } 85 \%\end{array}$ & $\begin{array}{l}\text { Nicht spezifisch für ein } \\
\text { bestimmtes Genus. Werte für } \\
\text { Candida sp. und Aspergillus sp. in } \\
\text { etwa vergleichbar. }\end{array}$ & {$[3,20]$} \\
\hline $\begin{array}{l}\text { Candida Mannan } \\
\text { und Antimannan }\end{array}$ & Candida & Serum & $83 \%$ & $86 \%$ & $\begin{array}{l}\text { Kombinierter Mannan- und } \\
\text { Antimannan-Nachweis deutlich }\end{array}$ & [19] \\
\hline
\end{tabular}




\begin{tabular}{|l|l|l|l|l|l|}
\hline $\begin{array}{l}\text { Latex- } \\
\text { Agglutination }\end{array}$ & Cryptococcus & $\begin{array}{l}\text { Serum, } \\
\text { Liquor }\end{array}$ & $93-100 \%$ & akkurater als Einzelnachweis. & \\
\hline $\begin{array}{l}\text { Cryptococcal } \\
\text { Antigen Lateral } \\
\text { Flow Assay }\end{array}$ & Cryptococcus & $\begin{array}{l}\text { Serum, } \\
\text { Liquor, } \\
\text { Urin }\end{array}$ & $\begin{array}{l}\text { Serum: 98 \% } \\
\text { Liquor: } 99 \% \\
\text { Urin: } 85 \%\end{array}$ & $\begin{array}{l}\text { Serum: 98 \% } \\
\text { Liquor: 99\% }\end{array}$ & [21] \\
\hline $\begin{array}{l}\text { Antigen } \\
\text { Nachweis }\end{array}$ & $\begin{array}{l}\text { Histoplasma, } \\
\text { Coccidioides, } \\
\text { Blastomyces }\end{array}$ & $\begin{array}{l}\text { Serum, } \\
\text { Liquor, } \\
\text { Urin, } \\
\text { BAL }\end{array}$ & $82-100 \%$ & $98-100 \%$ & Kreuzreaktionen möglich. \\
\hline $\begin{array}{l}\text { Antikörper- } \\
\text { Nachweis }\end{array}$ & $\begin{array}{l}\text { Histoplasma, } \\
\text { Coccidioides, } \\
\text { Blastomyces }\end{array}$ & Serum & $67-100 \%$ & - & 23] \\
\hline
\end{tabular}

* 1x; eine positive Probe, 2x; zwei aufeinanderfolgende positive Proben 
Abbildung 1: Fundament und Säulen der Pilzdiagnostik.

Abbildung 2: Direktmikroskopische Präparate. A) KOH-Präparat eines Sputums mit Aspergillus-Hyphen und Pseudohyphen von Candida albicans (Pfeil), B) KongorotPräparat einer Haarwurzel mit ektotrichem Sporenbefall (passend u.a. zu Microsporum canis), C) Silberfärbung eines histologischen Schnitts einer Hautbiopsie mit Pilzelementen von Sporothrix schenckii, D) PAS-Färbung eines Gewebes mit Hyphen bei einer Mukormykose mit Lichtheimia corymbifera. (Für die Bilder A und D bedanke ich mich bei Mirjam Grossenbacher.)

Abbildung 3: Algorithmus zur Inkubationszeit der Primärkultur in der Pilzdiagnostik. Nach der vorgeschlagenen Zeit kann eine Kultur als negativ berichtet werden, wenn keine Pilze gewachsen sind. Abbildung nach Bosshard PP [4].

Abbildung 4: Makro- und Mikromorphologie von Aspergillus fumigatus (A, B) und Microsporum canis (C, D). 